\title{
Losses of runaway electrons in MHD-active plasmas of the COMPASS tokamak
}

\author{
O Ficker ${ }^{1,2}$, J Mlynar ${ }^{1}, \mathbf{M}$ Vlainic $^{1,2,3}$, J Cerovsky ${ }^{1,2}, \mathbf{J ~ U r b a n}^{1}$, \\ P Vondracek $^{1,4}$, V Weinzettl ${ }^{1}$, E Macusova ${ }^{1}$, J Decker ${ }^{5}, \mathbf{M}$ \\ Gospodarczyk $^{5}$, P Martin ${ }^{7}$, E Nardon ${ }^{8}$, G Papp ${ }^{9}$, V V \\ Plyusnin $^{10}$, C Reux ${ }^{8}$, F Saint-Laurent ${ }^{8}$, C Sommariva ${ }^{8}$, J \\ Cavalier $^{1,11}$, J Havlicek ${ }^{1}$, A Havranek ${ }^{1,12}$, O Hronova ${ }^{1}$, M \\ Imrisek $^{1}$, T Markovic ${ }^{1,4}$, J Varju ${ }^{1}$, R Paprok ${ }^{1,4}, \mathbf{R}$ Panek $^{1}$, M \\ Hron $^{1}$ and the COMPASS team \\ ${ }^{1}$ Institute of Plasma Physics of the CAS, CZ-18200 Praha 8, Czech Republic \\ 2 FNSPE, Czech Technical University in Prague, CZ-11519 Praha 1, Czech Republic \\ ${ }^{3}$ Department of Applied Physics, Ghent University, 9000 Ghent, Belgium \\ ${ }^{4}$ FMP, Charles University, Ke Karlovu 3, CZ-12116 Praha 2, Czech Republic \\ ${ }^{5}$ Swiss Plasma Centre, EPFL, CH-1015 Lausanne, Switzerland \\ ${ }^{6}$ Universita' di Roma Tor Vergata, 00133 Roma, Italy \\ 7 Consorzio RFX, Corso Stati Uniti 4, 35127 Padova, Italy \\ ${ }^{8}$ CEA, IRFM, F-13108 Saint-Paul-lez-Durance, France \\ ${ }^{9}$ Max Planck Institute for Plasma Physics, Garching D-85748, Germany \\ ${ }^{10}$ Centro de Fusao Nuclear, IST, Lisbon, Portugal \\ ${ }^{11}$ Institut Jean Lamour IJL, Universite de Lorraine, 54000 Nancy, France \\ ${ }^{12}$ FEE, Czech Technical University in Prague, CZ-12000 Praha 2, Czech Republic \\ E-mail: ficker@ipp.cas.cz
}

\begin{abstract}
Significant role of magnetic perturbations in mitigation and losses of Runaway Electrons (REs) was documented in dedicated experimental studies of RE at the COMPASS tokamak. RE in COMPASS are produced both in low density quiescent discharges and in disruptions triggered by massive gas injection (MGI). The role of the $\mathrm{RE}$ seed produced in the beginning of the discharge on the subsequent RE population proved significant. Modulation of the RE losses by MHD instabilities was observed at several characteristic frequencies as well as by magnetic field oscillations related to power supplies. Magnetic islands seem to suppress the losses as the HXR signal is low and coherent with the island rotation frequency. Moreover, periods of increased losses of REs observed in the current quench (CQ) and early RE beam plateau phase of the MGI disruptions seem to be linked to the bursts of magnetic perturbation and to the observation of filaments in the fast visible camera images.
\end{abstract}

Keywords: tokamaks, runaway electrons, MHD instabilities, disruptions 


\section{Introduction}

Control and/or mitigation of Runaway Electrons (REs) present one of the key tasks for experimental work of present tokamaks in support of the ITER programme. Indeed, models of ITER disruption evolution predict generation of RE beam with several tens of $\mathrm{MeV}$ to carry up to $70 \%$ of pre-disruptive plasma current [T]. As the deposition of the RE current can be highly localised, the deposited energy could severely damage plasma facing components and blanket modules of ITER. Following previous experiments [2] and recent modelling efforts [3] it appears that improved understanding of the link between the complex dynamics of evolution of perturbed magnetic surfaces during the disruption and the RE generation and losses present one of the key contributions towards safe operation of ITER. However, this task is quite challenging due to short timescales and significant radiation loads that can lead to saturation or degradation many of diagnostic signals. The equilibrium reconstruction, e. g. EFIT that is applied to COMPASS discharges, is also ruled out for a fast discharge termination. In this respect it is essential to understand the response of REs to magnetic perturbations under controlled conditions, i.e. in the current flat-top phase of the discharge. Subsequently, the acquired knowledge can be extended towards plasma disruption timescales with support of the relevant MHD codes (e.g. JOREK [4]).

The COMPASS tokamak [5, 6] has been systematically contributing to dedicated RE research from 2014. It is an experimental device with ITER-like plasma cross-section, major radius $R_{0}=0.56 \mathrm{~m}$ and minor radius $a=0.23 \mathrm{~m}$. The typical toroidal field is $B_{T}=1.2 \mathrm{~T}$ and plasma current in the flat-top phase is $I_{p}<400 \mathrm{kA}$. The COMPASS plasmas can be operated in both limiter and divertor configuration, the latter allowing for routine H-mode operation [5]. Neutral beam heating at $40 \mathrm{keV}$ can inject up to (600 $\mathrm{kW}$ ) of additional power. The main advantages of the machine are its flexibility and low operational costs. Population of REs is observed in COMPASS even at relatively high plasma densities $n_{\mathrm{e}} \sim 4-6.10^{19} \mathrm{~m}^{-3}$, with a strong dependence on the gas fuelling scenario in the initial phase as well as on the plasma shape [7]. The COMPASS experiments are normally deuterium fuelled and the typical pulse length is about $0.4 \mathrm{~s}$, although the low current circular discharge with high fraction of REs can last up to one second [8]. The disruptive scenario with RE beam generation following argon puff was achieved in COMPASS [9] and currently it presents an important topic for further investigation.

\subsection{MHD and RE related Diagnostics}

The COMPASS tokamak features a rich set of magnetic diagnostics [10], allowing for rather detailed measurements of both poloidal and toroidal characteristics of magnetic perturbation in the plasma, including magnetic islands and TAEs (Toroidal Alfvn

Eigenmodes). It consists of three poloidal arrays of internal Mirnov (pick-up) coils (MC) at different toroidal positions covering all three components of magnetic field $(r, \theta, \phi)$ by 24 coils each (i.e. $3 \times 3 \times 24$ coils in total), see figure $\mathbb{\text { ⿴囗 }}$. Furthermore, 16 
Internal Partial Rogowski coils (IPR) circumventing poloidal cross section (so that their combined signal provides a direct measure of the plasma current), several flux loops and extensive number of saddle coils may be used in studies of magnetic configuration and perturbations. For the analysis reported further in this paper, namely the outer midplane (OMP) coils of all arrays have been used.

The primary means of the RE research at COMPASS relies on standard hard X-ray (HXR) diagnostics based on a pair of $\mathrm{NaI}(\mathrm{Tl})$ scintillation detectors with photomultiplier tube (PMT) and a composite scintillator (EJ410 - ZnS(Ag) embedded in a plastic matrix) with PMT shielded by $10 \mathrm{~cm}$ of lead. Although the latter detector features an enhanced sensitivity to photoneutrons, the experience at COMPASS tokamak demonstrated that the relative intensity of HXR is sufficiently high to overrun the neutron signal and make the main component of the $\mathrm{ZnS}(\mathrm{Ag})$ data in most of the $\mathrm{RE}$ dedicated experiments. Therefore, in the following it will be referred to as the shielded HXR detector, sensitive to HXR energies of approx. $500 \mathrm{keV}$ and above. The energy range of the unshielded $\mathrm{NaI}(\mathrm{Tl})$ scintillation detectors starts approximately at $50 \mathrm{keV}$. Due to high intensity of the HXR signal in the RE experiments - note that there is no collimation - the detectors typically work in the current mode operation. The approximate distance of both detectors from the tokamak main axis is $4 \mathrm{~m}$, for their location see figure 2 .

Plasma density was monitored and controlled using the 2-mm interferometer with central vertical line of sight. In recent experimental campaigns the fast IR camera Telops FASTIR $2 \mathrm{~K}$ was located on tangential port in the direct view of the OMP protection limiter (made of graphite). The camera was used for studies of first wall heat loading due to the RE losses, which were monitored with frame-rates up to $30 \mathrm{kfps}$ and spatial resolution $\sim 1 \mathrm{~mm} /$ pixel. Besides, for investigation of the MGI induced disruptions with runaway beam generation, the fast visible camera Photron Mini UX100 proved to be very useful. The camera is situated at the midplane and covers a wide angle at standard frame-rate of $8 \mathrm{kfps}$.

\subsection{RE generation control via plasma density}

Based on the previous experience [G], the RE seed generated in the plasma current ramp up phase proves to be well controlled by the plasma fuelling just before the plasma breakdown and in the initial $10 \mathrm{~ms}$ of the discharge. Without the RE seed, the RE generation in the current flat-top phase appears only at very low plasma densities, approximately $n_{\mathrm{e}}<1.5 \cdot 10^{19} \mathrm{~m}^{-3}$ that corresponds to $E / E_{c} \geq 10$ where $E_{c}=\frac{e^{3} n_{\mathrm{e}} \ln \Lambda}{4 \pi \epsilon_{0}^{2} m_{\mathrm{e}} c^{2}}$ and $E$ is an estimate of the electric field inferred from the loop voltage measurement. Once the RE seed is present, the RE confinement is much better in the divertor configuration and in the elongated limited plasma compared to the circular limited plasma [7]. Indeed, in the circular limited configuration it suffices to increase the density to $n_{\mathrm{e}}=3 \cdot 10^{19} \mathrm{~m}^{-3}$ in order to initiate relatively fast decay of the $\mathrm{RE}$ population. 

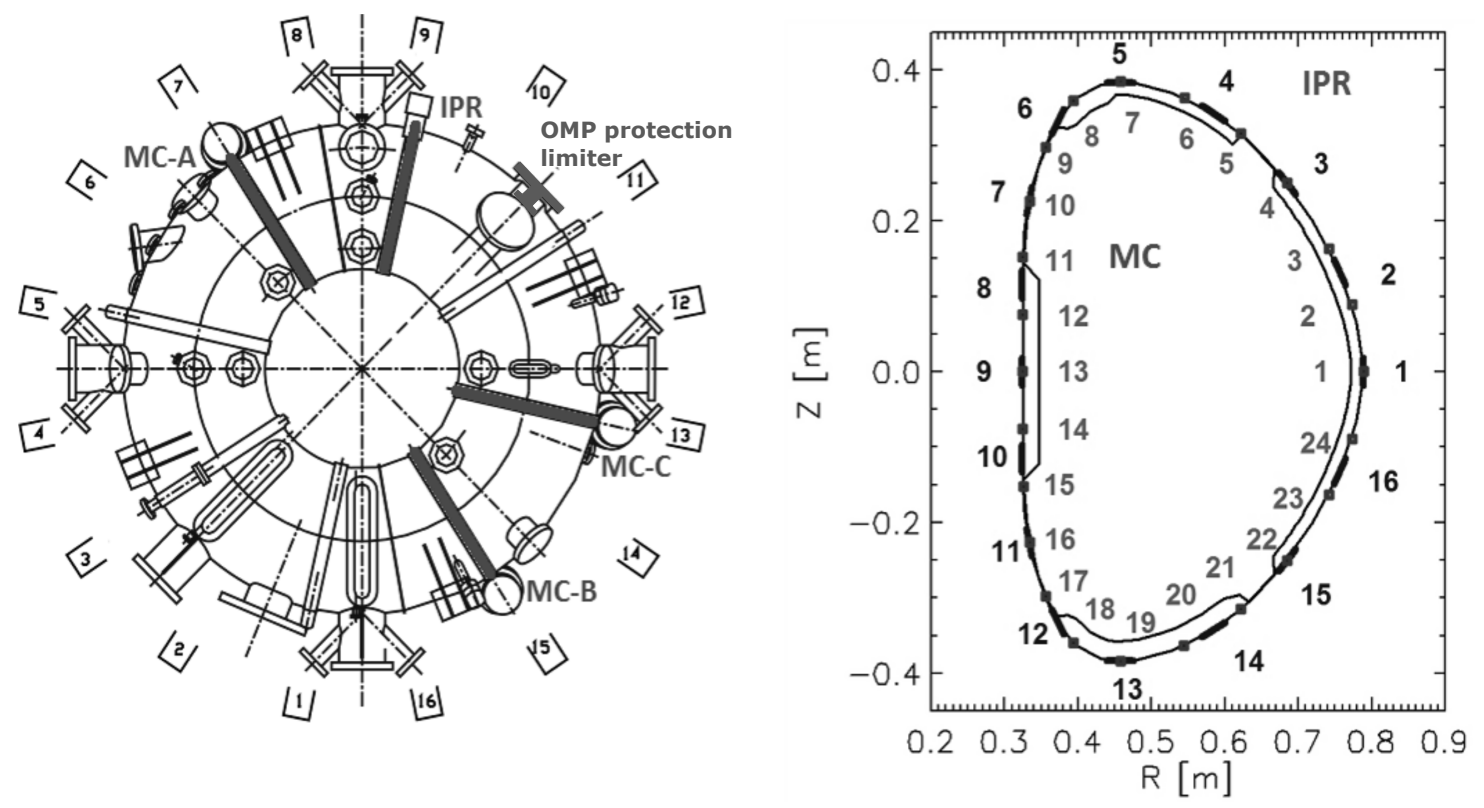

Figure 1. The COMPASS tokamak, left: top view with toroidal positions of the MHD coil arrays (all located inside the vacuum vessel), limiter position corresponding to section [2.2, right: vaccum vessel poloidal cross-section with the distribution of MC and IPR coils.

The next sections accentuate the influence of the background plasma and external coil current oscillations on the runaway electron population and are organised as follows: in section 2$]$ the effect of ST instability on RE in discharges with controlled RE population is presented, section [2.2] outlines the effect of magnetic islands on RE losses, section [3] details direct (B.]) and indirect (3.2) effect of poloidal field power supply oscillations and in part $⿴$ the interplay of magnetic perturbations and $\mathrm{RE}$ losses during the disruptions is reported.

\section{RE losses due to MHD instabilities}

\subsection{Increased losses during sawtooth crashes}

A comparison of a series of similar discharges with a rather high flat-top density $\left(n_{\mathrm{e}} \sim\right.$ $6 \cdot 10^{19} \mathrm{~m}^{-3}$ ) in the divertor configuration was carried out, in order to better understand the influence of different plasma fuelling waveforms on the runaway electron population. Furthermore, the discharges were characterised by a relatively high frequency sawtooth instability $(2 \mathrm{~ms})$. It can be concluded that the changes in the magnetic topology and induced radial plasma flow substantially increase the RE losses. Note that from the measured data it cannot be inferred whether REs from the core or rather from the edge are lost in the crashes as sawtooth instability is know to strongly affect edge physics in COMPASS[M]. The sawtooth instability presents an especially important case with respect to its potential to generate a population of energetic electrons due to the local 


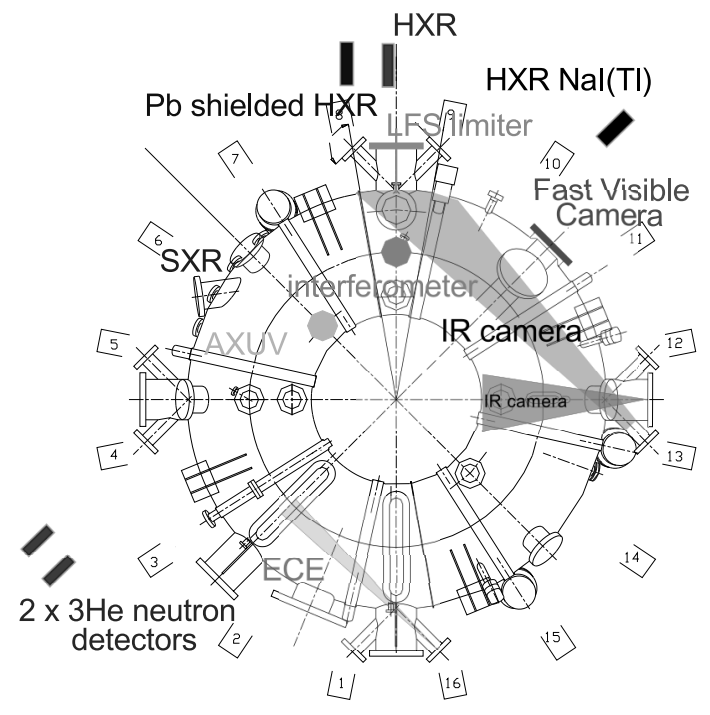

Figure 2. The most recent layout of the RE-related diagnostics.

electric field induced in fast reconnection theoretically considered and even observed in TCV [1.2]. Although it seems unlikely that such a population could be confined for a sufficient time in order to become a RE seed and eventually lead to a significant RE current, this generation source was investigated during COMPASS fuelling experiments. The result is negative at the detection level of the utilised HXR detectors, see figure B. In the discharge where the initial fuelling and thus also the density were kept at higher levels to prevent RE seed generation (\#8634), negligible HXR signal was observed during the current flat-top even though the sawtooth instability showed the same behaviour as in the reference discharge with the same initial parameters except the initial fuelling (\#8636) where the HXR signal was clearly correlated with sawtooth crashes. This result has proved to be reproducible. No clear correlation between the size of the sawtooth crash or the period of the instability and the size of HXR peaks was identified so far.

\subsection{RE losses and magnetic islands}

Magnetic islands appear often in COMPASS plasmas, depending on the safety factor profile $q(r)$ [1:3]. The oscillations of the pick-up coil signal that are caused by the rotating magnetic island may be identified using a time-resolved frequency analysis. In COMPASS discharges without auxiliary heating, the typical frequency of rotation of stable magnetic islands with toroidal mode number $n=1$ is $8-10 \mathrm{kHz}$. This section is focused on the discharges with plasma densities $n_{\mathrm{e}}$ from 1 to $3 \cdot 10^{19} \mathrm{~m}^{-3}$ and a rather high loop voltage. In these discharges, REs are present while carrying just a small fraction of plasma current and the interaction of REs with the background plasma can be

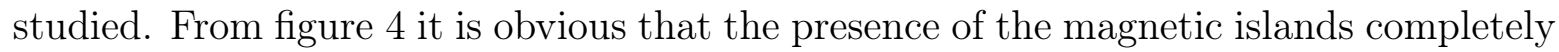
alters the dynamics of the RE losses compared to a MHD-quiescent plasma discharges. It seems that the magnetic islands can behave as a barrier for REs and the losses are 


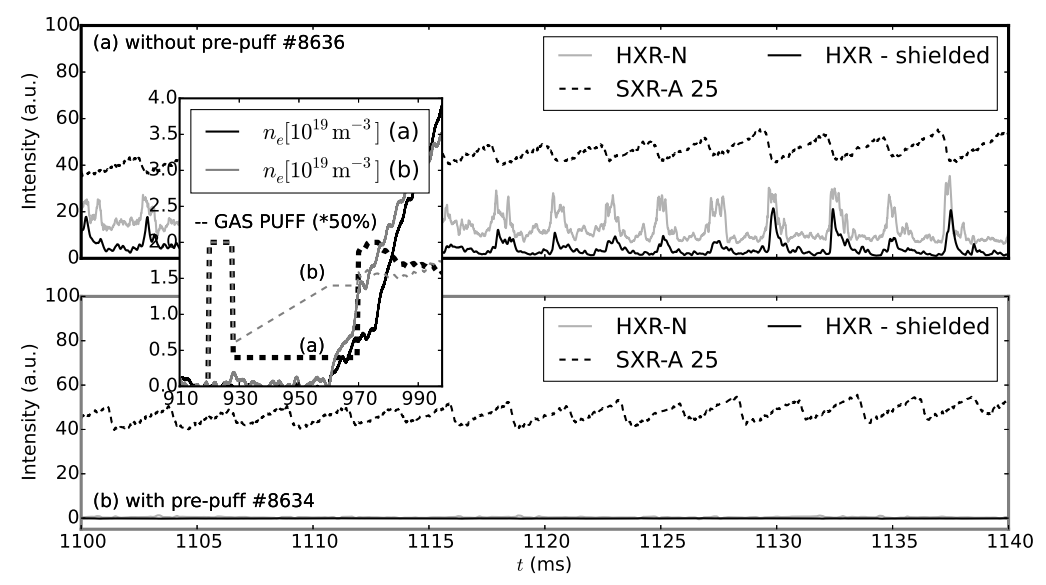

Figure 3. The initial fuelling and density evolution just after the plasma breakdown (inserted image) for two otherwise identical discharges where REs are affected by the sawtooth instability. Increase of the initial fuelling results in a strong suppression of RE seed and subsequently no HXR burst connected to sawtooth crashes are observed.

significantly lowered until the end of the discharge when the rest of the RE population is released. The density as well as the initial fuelling was kept the same in each pair of the presented discharges $\left(n_{e}=1.5 \cdot 10^{19} \mathrm{~m}^{-3}\right.$ in $\# 10004$ and $\# 10006, n_{e}=1.8 \cdot 10^{19} \mathrm{~m}^{-3}$ in $\# 13084$ and $\# 13085$ ). Furthermore, the measured loop voltage in the discharges with magnetic islands is significantly lower compared to the quiescent discharges. This supports the hypothesis of a decreased radial transport of REs due to magnetic islands. Besides, the intentional decreases in the plasma current seem to affect the RE losses much more in the discharges with perturbed topology of magnetic surfaces. In order to completely understand the effect of magnetic islands on REs, the role of the size, position and rotation frequency of magnetic islands must be studied and compared with MHD models, e.g. JOREK.

Observation of oscillations in the HXR signal intensity with frequencies similar to the frequency of island rotation was first briefly reported in [13]. The interplay between the HXR data and the magnetic data was later confirmed in dedicated COMPASS RE campaigns in discharges with a measurable population of REs and with magnetic oscillations of a sufficient amplitude. From these measurements, it can be concluded that the magnetic islands considerably influence confinement of REs at the plasma edge. Indeed, the coherence diagram in figure 5 shows that the HXR oscillations follow the changes of the island rotation frequency. The changes were induced by small decreases in the plasma current waveform. Furthermore, the second harmonic - practically undetectable in the simple spectrogram calculated from the Mirnov coil signal - appears in the coherence diagram.

In order to investigate whether the coherent RE losses correspond to the O-point or the $\mathrm{X}$-point of the magnetic island - in simplified terms, whether the HXR intensity is in phase or in counter phase to the magnetic oscillations - a method was developed using 
the available data, i.e. the non-localised HXR measurements and the signals from the rich and localised magnetic diagnostics on COMPASS. The presented method is based on the assumption that most of the REs are lost to the OMP protection limiter and the magnetic islands have toroidal mode number $n=1$, although the method might be modified for higher toroidal mode numbers. The method is using the cross-correlation that helps to identify the time delays between similar signals, in our case the magnetic fluctuation signals and the HXR signal (reference). The cross-correlation is more efficient than simple comparison of signals as it highlights common components of the time series. Before applying the cross-correlation analysis, the signals were band-pass filtered $\left(f_{l}=100 \mathrm{~Hz}, f_{u}=100 \mathrm{kHz}\right)$. An example of the delays derived from the crosscorrelation analysis for one segment of the signals for the discharge \#10004 is shown in the left part of figure [6. The time evolution of the cross-correlation delays $\tau$ is then shown in the right part of figure 6 (for each time the delays are detected as the left part of the figure shows). The changes in the frequency of the island rotation appear in this figure again and further support our assumptions. The time delays may be transformed to the toroidal phase shifts by a multiplication with the instant value of the angular frequency but in such a case the changes in the time evolution (due to the plasma current variation in $\# 10004$ ) would not be visible anymore. From the analysis of the phase

shifts between the integrated signals of the OMP coils of the 4 poloidal arrays (for the set-up see figure () it can be concluded that the enhanced RE losses correspond to the O-point of the magnetic islands.

Similar results were achieved also in FTU using the Cherenkov detector [14] (localised measurement of the RE losses, recently tested on COMPASS as well), for the detailed analysis see [15]. However, this technique is not fully reproducible for all COMPASS discharges due to multiple reasons, including a significant presence of higher toroidal mode component, saturation in the HXR data and also a limited validity of the key assumption that the HXR intensity oscillations originate in the OMP limiter. Note that a significant damage from the REs was also observed on the HFS. Nevertheless, these results highlight the importance of the edge magnetic topology for RE losses.

\section{Effects of the poloidal magnetic field power source oscillations on the RE losses}

In the COMPASS experiments, it turned out that RE losses are not modulated just by the plasma MHD instabilities but also by other frequencies linked to the power supplies (PS) of the magnetic field. The main power supplies of the COMPASS tokamak consist of two flywheel generators (FWG) and a set of thyristor $\mathrm{AC} / \mathrm{DC}$ converters [6, [6]. For the experiments presented in this part only one FWG was used with stored energy up to 45 MJ. The FWG typically rotates at 1400 RPM at the beginning of the discharge, the rotation frequency decreases approximately by 100-200 RPM towards the end of the discharge. The poloidal field PS use 12-pulse converters while the toroidal field PS 

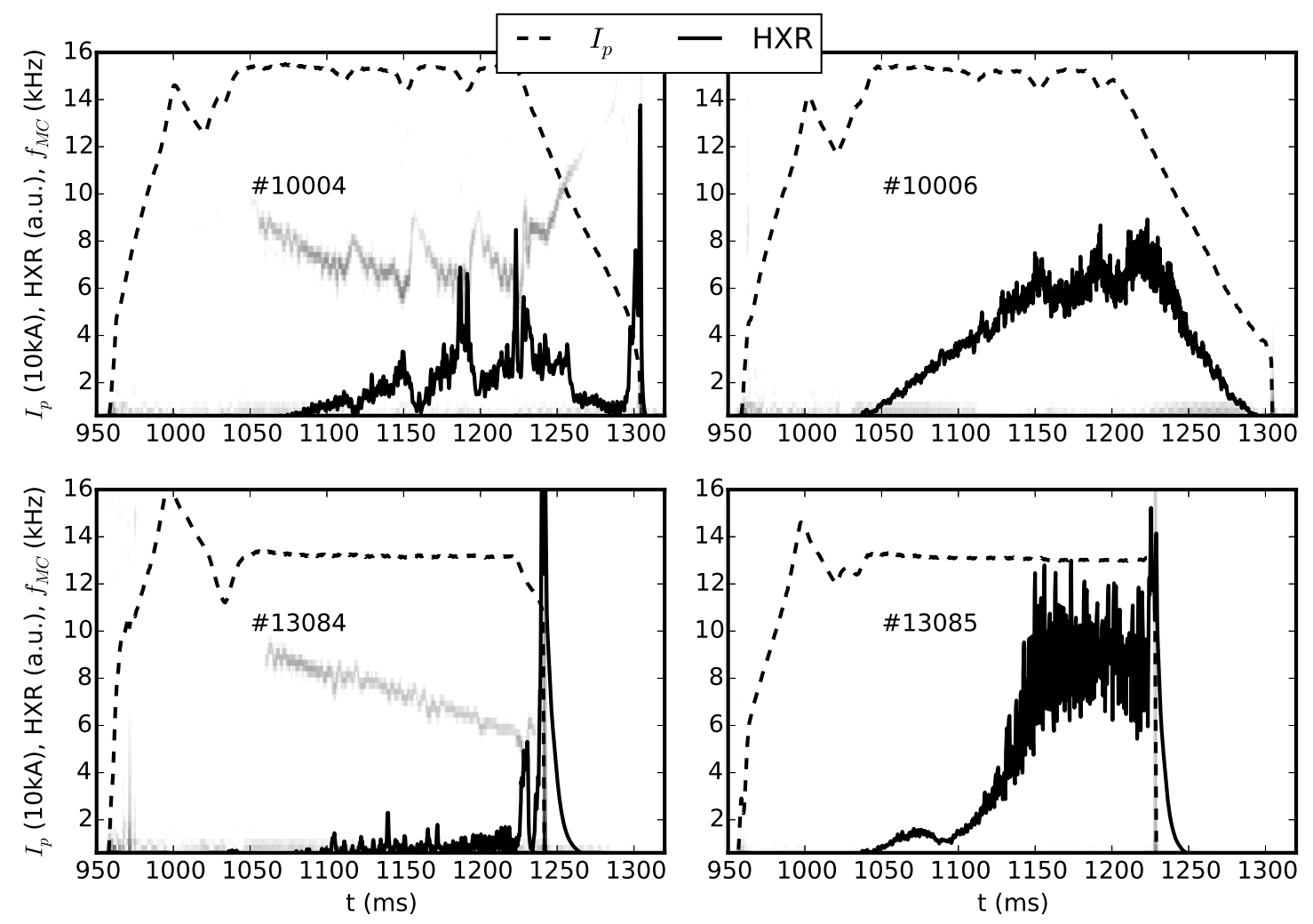

Figure 4. Comparison of two pairs of almost identical COMPASS discharges (the top pair differ in elongation \#10004: $\epsilon=1.0$ and \#10006: $\epsilon=1.1$, the discharges of the second pair were using identical waveforms for all pre-set parameters). Spectrogram of outer midplane Mirnov coil is in the background (grey), plasma current $I_{p}$ in a dashed line and signal of shielded HXR/PN detector in solid, the scale is the same in all frames.

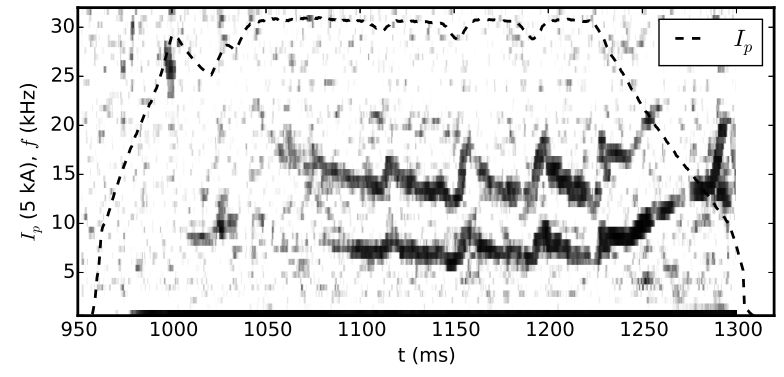

Figure 5. A coherence diagram of the Mirnov coil signal and the signal of the HXR detector together with the time evolution of the plasma current (dashed line), COMPASS discharge \#10004. The magnetic island rotation clearly correlates with the modulated HXR signal visible at approx. $8 \mathrm{kHz}$ and with the second harmonic. 

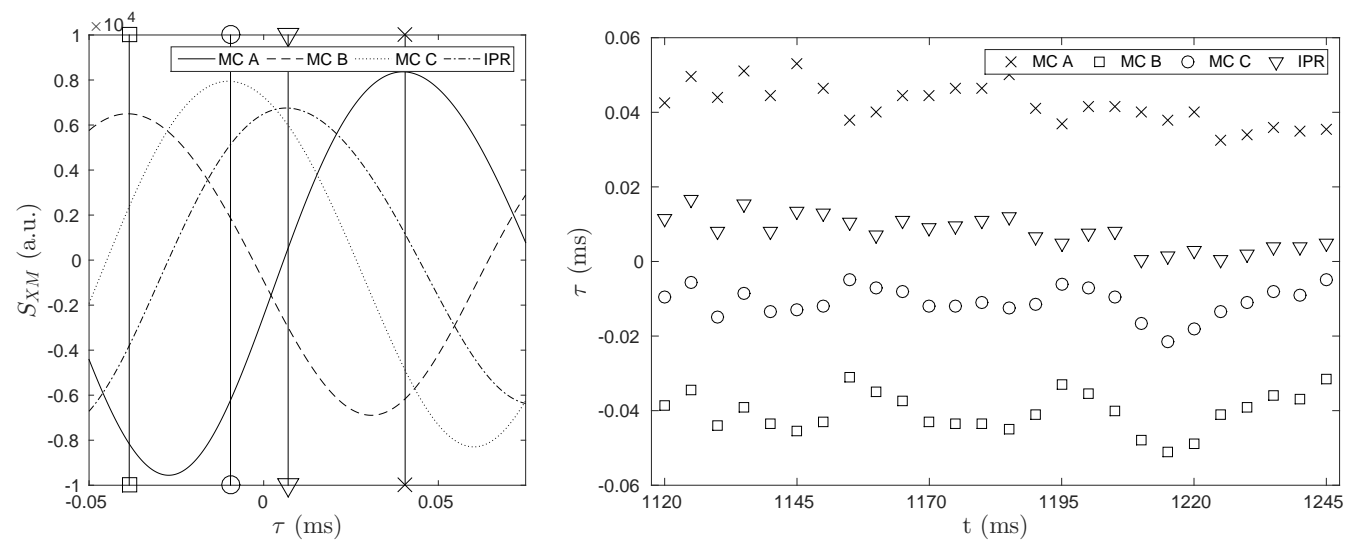

Figure 6. Left: An example of delays derived from cross-correlation analysis between the reference HXR data and the data from magnetic coils located at four different

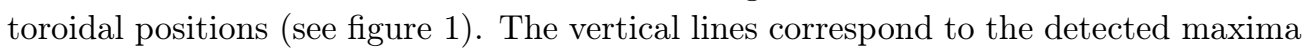
of the cross-correlation functions $S_{X M}, \tau$ is the lag (delay) of the cross-correlation functions. Right: time evolution of the lag, demonstrating that the RE losses culminate when the maximum of the field perturbation passes the OMP limiter.

use 24-pulse converters. Due to these technical constraints, the current in the poloidal field windings - and therefore the measured signals of the loop voltage, the equilibrium poloidal field and the shaping poloidal field - exhibit an oscillating component with a typical frequency of approx. $800 \mathrm{~Hz}$, while in the case of the toroidal field, the frequency is doubled. The effect of these PS oscillations on the COMPASS plasma is in general negligible, however it becomes very important in a case of sensitive phenomena, e.g. RE losses.

\subsection{Direct effect due to radial position change}

Small oscillations in the radial position of the order of several millimetres with frequency approximately $400 \mathrm{~Hz}$ appear in COMPASS as a reaction of the real-time position control (PID controller) to the above mentioned PS oscillations. These oscillations modulate the losses of REs namely in discharges with large RE population. Modulation of the RE losses by radial position was recently confirmed by the fast IR camera data that correspond to heat flux on the OMP protection limiter [17], see figure 7 . The left part of the figure also indicates that the increasing heat flux comes primarily from the growing RE population: the heat flux increases with the decreasing electron density, while the plasma temperature does not change significantly according to the Thomson scattering measurement. Moreover, in the discharges where intentional radial movements were applied [8] it was shown that the losses might be increased also by slow outward movement. These results indicate that namely the losses of the runaway electrons from the very edge of plasma are modulated by this mechanism. 

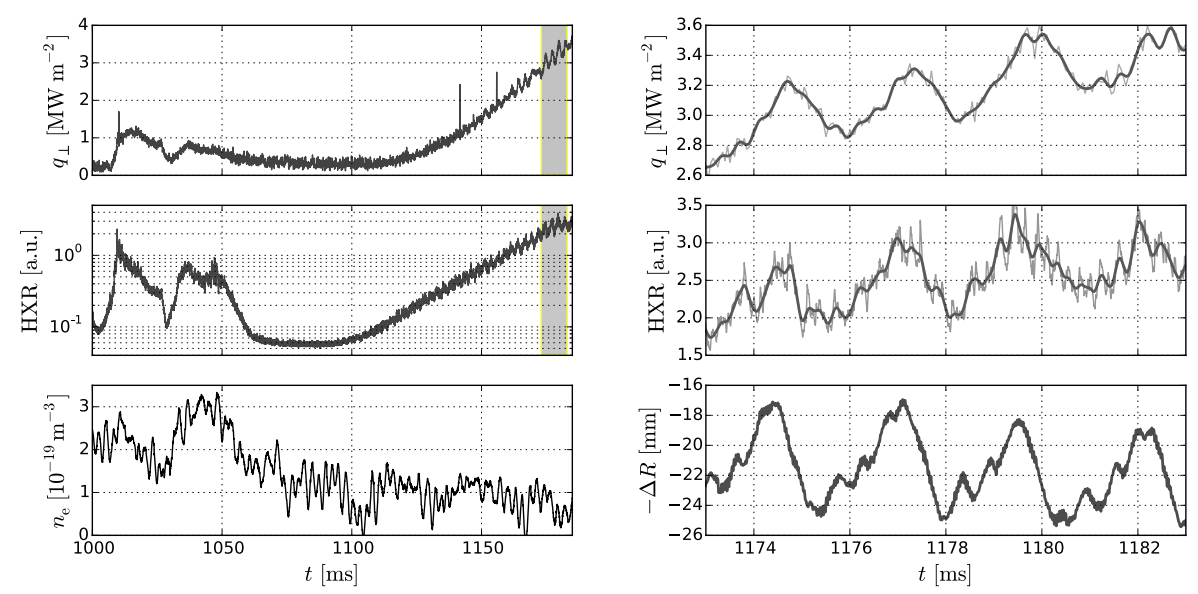

Figure 7. Left: Time evolution of the heat flux $q_{\perp}$ calculated from the fast IR camera data, HXR signal and the electron density $n_{\mathrm{e}}$ as measured in the COMPASS discharge with strong RE generation (\#12084). Right: The detail of the highlighted region including the oscillation of the radial positon of the magnetic axis as calculated by EFIT.

\subsection{Indirect effect due to interaction with plasma MHD perturbations}

The radial oscillations of the plasma position do not present the only occurrence of an interplay between the RE losses and the PS oscillations. Interestingly, in case of smaller RE population, very pronounced periodic peaks with a frequency close to $1 \mathrm{kHz}$ were observed in the signals of all HXR detectors and even in the form of bursts of saturated pixels in the fast visible camera images. In order to determine whether this phenomenon was connected to the power source oscillations, the initial frequency of the FWG was changed in a series of almost identical discharges. Comparison of a pair of these discharges is presented in figure $\mathbf{8}$. Indeed, the results demonstrate that the change in the FWG rotation frequency (which drives the oscillations of the loop voltage signal) causes a proportional change of the frequency of the HXR intensity peaks.

The fact that the HXR peak frequency in this regime of RE losses changes when the FWG rotation frequency is changed presents a very robust result. However, it was also clearly observed that in different discharge scenarios the frequency of the HXR periodic peaks may have different values with the same FWG frequency. Therefore, 14 relevant discharges were systematically analysed in order to determine the key parameters behind this phenomenon. It has been found that the frequency of the losses show significant dependence on the value of the edge safety factor $q_{95}$ : the lower the $q_{95}$, the higher the frequency of the HXR peaks. In order to cancel the dependence on the FWG frequency, figure 9 gives the ratio of the HXR and the loop voltage oscillation frequencies as a function of $q_{95}$. Although it seems that the value of the studied ratio may reach any number in the continuous interval, the values of the ratio could be primarily only rational numbers (e.g. $\{1,3 / 2,2\}$ ) with the scatter being caused by different effects.

It is worth noting that a weak magnetic structure, probably a small magnetic island, 

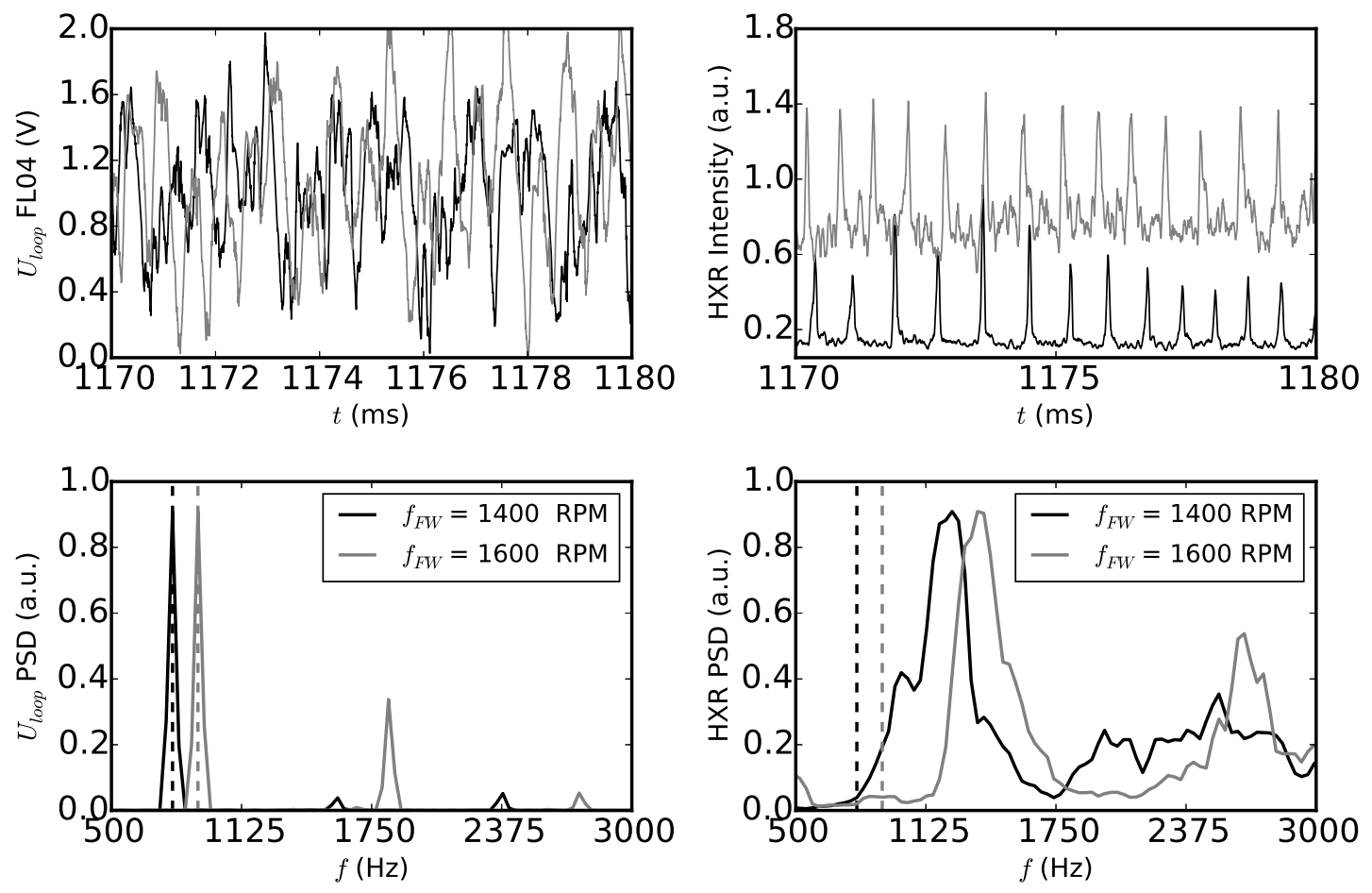

Figure 8. Comparison of the loop voltage (left) and HXR (right) oscillations in the two discharges with different FWG rotation frequencies (1400 RPM in the discharge \#10874, 1600 RPM in the discharge \#10875). Time traces of the signal (top) and the frequency analysis (bottom). Vertical lines correspond to oscillations in the loop voltage signal in both graphs).

can be observed in the spectrogram of the outer mid-plane diagnostic coils at $6-10 \mathrm{kHz}$ during this type of the RE losses (see figure ए0). Based on these observations a proposed explanation of the HXR peak frequencies is a resonant interaction of the power source oscillations with weak magnetic islands. This hypothesis can explain the weak trace in the spectrogram and the transitions from this mechanism of the RE losses to the mechanism dominated by large magnetic islands that was described in the previous section. Provided that the magnetic islands behave like a barrier for REs (see section [2.2), this mechanism may correspond to a repeating destruction of such barrier. Details of the interaction between the PS oscillations and the magnetic islands, dependent on their mode number, are yet to be disclosed.

\section{RE losses and MHD activity during current quench and beam plateau phase}

The wide variety of periodic RE losses phenomena identified in quiescent discharges and described in the previous text shows that even minor changes in the topology of magnetic surfaces may critically affect RE losses. This presents an important conclusion for the generation of runaway electron beam during a massive gas injection (MGI) 


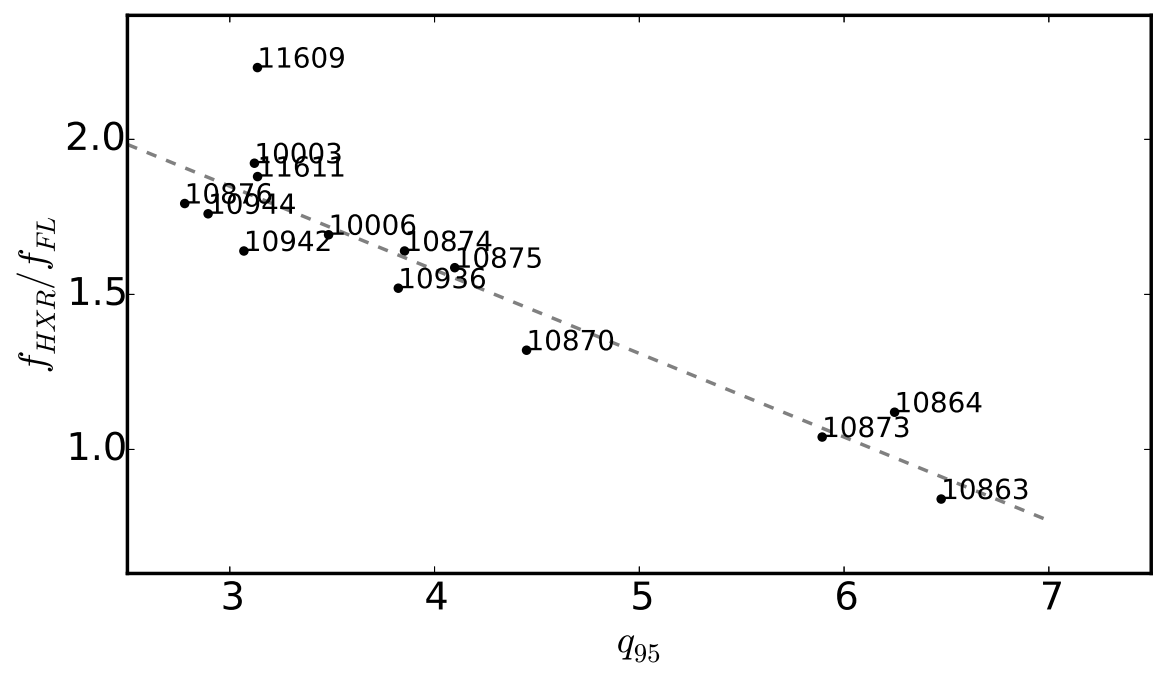

Figure 9. The dependence of the ratio of the frequency of periodic peaks of HXR signal $f_{\mathrm{HXR}}$ and the dominant frequency of the poloidal field oscillations as measured by the flux loop $f_{\mathrm{FL}}$ on the safety factor value at the edge $q_{95}$. Discharge numbers are shown as well.

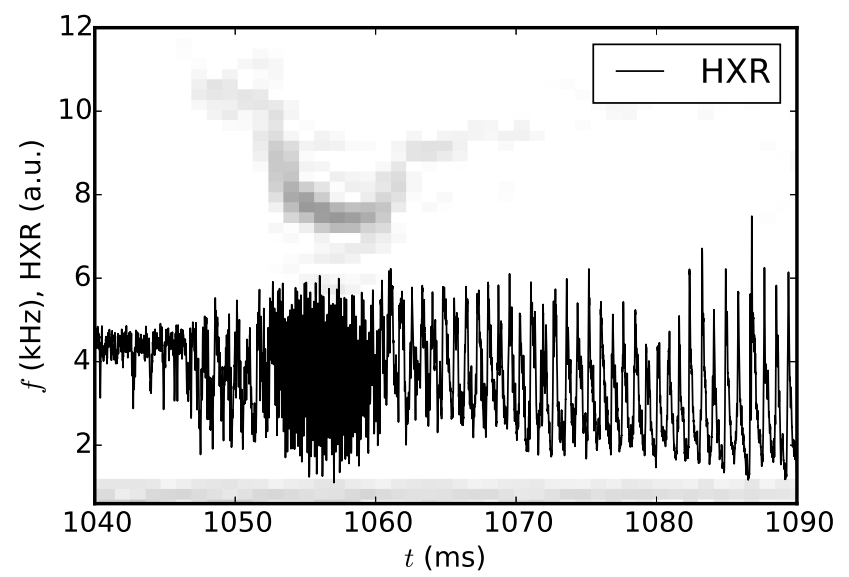

Figure 10. Spectrogram of the OMP IPR coil signal and the HXR intensity signal for discharge \#10945, three regimes of RE losses may be identified - aperiodic regime in the very begining, periodic peaks with frequency above $1 \mathrm{kHz}$ accompanied by a weak trace in the spectrogram and $8 \mathrm{kHz}$ losses coherent with the magnetic island. 
triggered or mitigated disruption. Generation of the post-disruptive beams has been systematically studied on many large and medium size tokamaks [20, 2:3, 32, 31], while the most thorough analysis of the interplay between the magnetic perturbation level and RE beam current was performed at TEXTOR [2, 30]. The scenario was also developed and studied on COMPASS [9]. A typical COMPASS post-disruption RE beam is generated in a MGI-triggered (argon) disruption in the current ramp-up phase of circular limited discharges at 12-25 ms after the plasma breakdown. Argon pressure in the range $0.8-2.8$ bar was used with the number of injected particles being in the order of $10^{19}-10^{20}[9]$. The radial stability of the plasma during the disruption and of the subsequent beam as well as sufficient control of the initial density present the main challenges in these experiments on COMPASS and for the statistical analysis. The COMPASS post-disruption RE beams were obtained in $B_{t}=1.15 \mathrm{~T}$, thus it seems that there is a different threshold for the beam generation than the one found on TEXTOR ([2], $B_{t} \geq 2 \mathrm{~T}$ required for RE beam generation). The large current spike typically occurring during the disruption is replaced by multiple smaller events in the case of MGI disruption on COMPASS, see the evolution of $I_{p}$ in figure $\mathbb{W}$. These events are always accompanied by a negative loop voltage peak, bursts of magnetic perturbations and usually also by HXR spikes. This character of the CQ phase is common for both disruptions with and without the RE beam generation. In figure $\square$ a comparison of the two cases is displayed: multiple MHD bursts (strongest at the HFS - MC C13 signal is displayed in the graphs) often accompanied by HXR spikes occur in both cases however the perturbations are smaller in the case when the RE beam is generated ( \#12190). The magnetic fluctuations then almost disappear and the HXR signal becomes rather continuous while in the case of the other discharge (\#12189) the fluctuations continue until the plasma current disappears. The dependence of the post-disruptive RE beam current on the level of magnetic perturbations was statistically analysed for COMPASS MGI disruptions. However, no clear threshold value nor a trend was found as the discharges conducted in different campaigns are very hard to compare, partly due to the position instability and different distance from the Mirnov coils. On the other hand, the level of magnetic perturbation displayed in figure $\mathbb{U}$ is close to the theoretically predicted threshold value where perturbations should prevent the RE beam generation $\left(\delta B / B_{t}>10^{-3},[24]\right)$ and the two subsequent discharges displayed in figure $\square$ follow the expected trend.

The disruption evolution was recently monitored also by a fast visible radiation camera (8kfps). During the studied disruptions, the camera detects namely the line radiation of argon. In figure 12, four selected frames are displayed: in the top left frame Ar radiates at the plasma edge close to the MGI valve just after the injection, top right frame shows thick helical structures in the current quench when argon is already toroidally distributed. In the bottom left frame the thin filamentary structures appear within the phase where $I_{p}$ stops decreasing and minor bursts of magnetic perturbation still occur. The last, bottom right frame displays the late post-disruptive RE beam phase that seems to be very homogenous. 

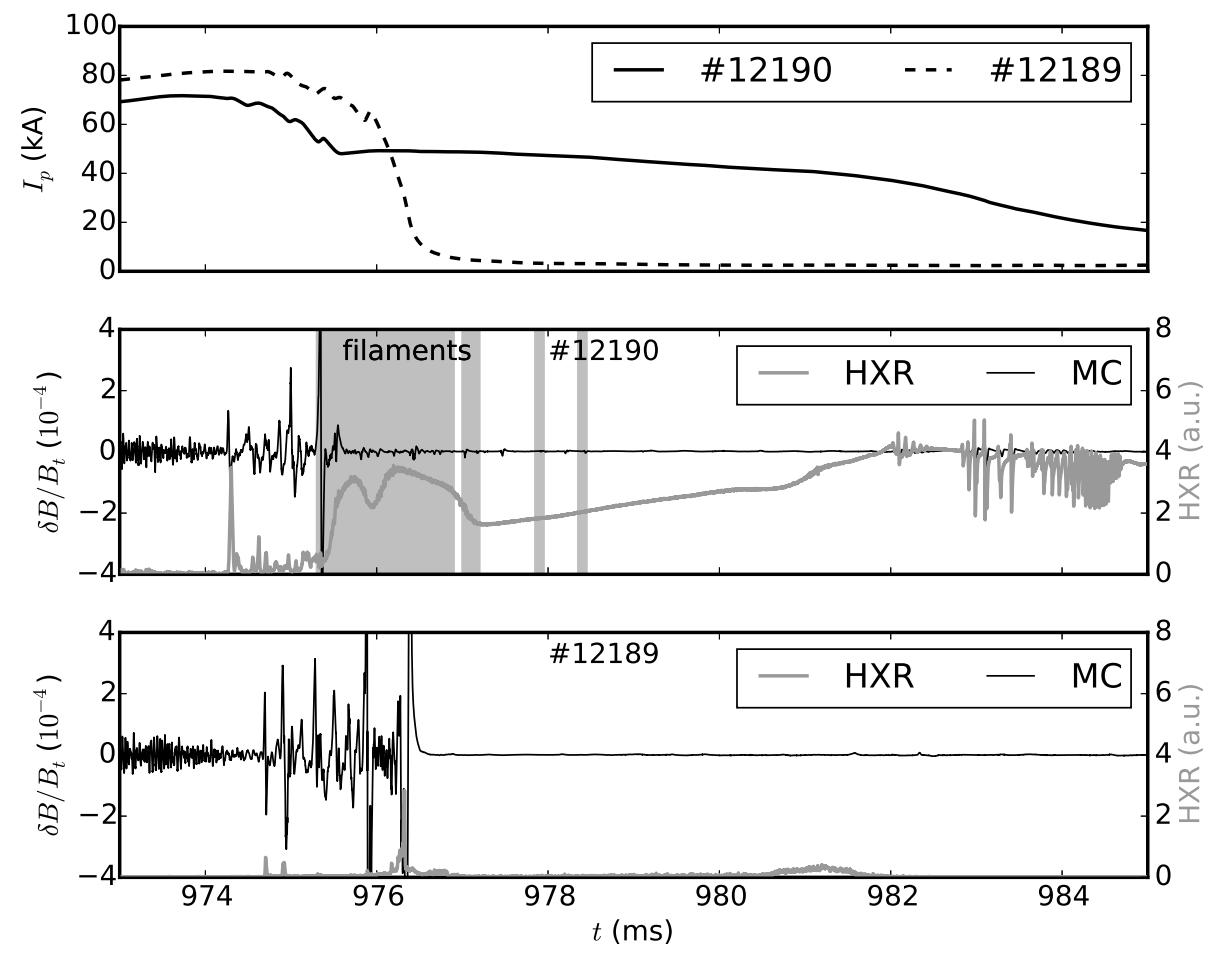

Figure 11. The detail of the current quench and early RE plateau phase of the discharges \#12189 (no beam) and \#12190 (beam produced), comparison of magnetic fluctuation level measured by HFS Mirnov coil and HXR signal, the scale of signals is the same in both subplots. Grey areas represent the frames where the filaments were detected.

The filaments in the early beam phase are accompanied by increased HXR signal (see the marked time range in figure $\square$ ) that seems to be almost continuous and tend to decrease after the main filamentary phase. Later in the beam phase, isolated filaments may be still observed but the Mirnov coil data are generally quiescent. The frames with the filaments can be linked to the small bursts of magnetic turbulence, however, the camera might miss some of the filaments and measurements using higher frame rate are necessary in order to confirm the correlation. The post-disruptive RE beam typically terminates at the LFS as the control system fails to prevent the radial expansion. Furthermore, in the particular discharge \#12190 the faster dissipation of the beam current is accompanied by large periodic HXR spikes (see figure $\square 2$ from $t=982 \mathrm{~ms}$ ) and small magnetic perturbation bursts. Relatively large measured radial position of the beam at this phase indicates that this event occurs due to the interaction of the beam with the protection limiter. Both the filamentary phase and the beam dissipation are definitely worth further study. 


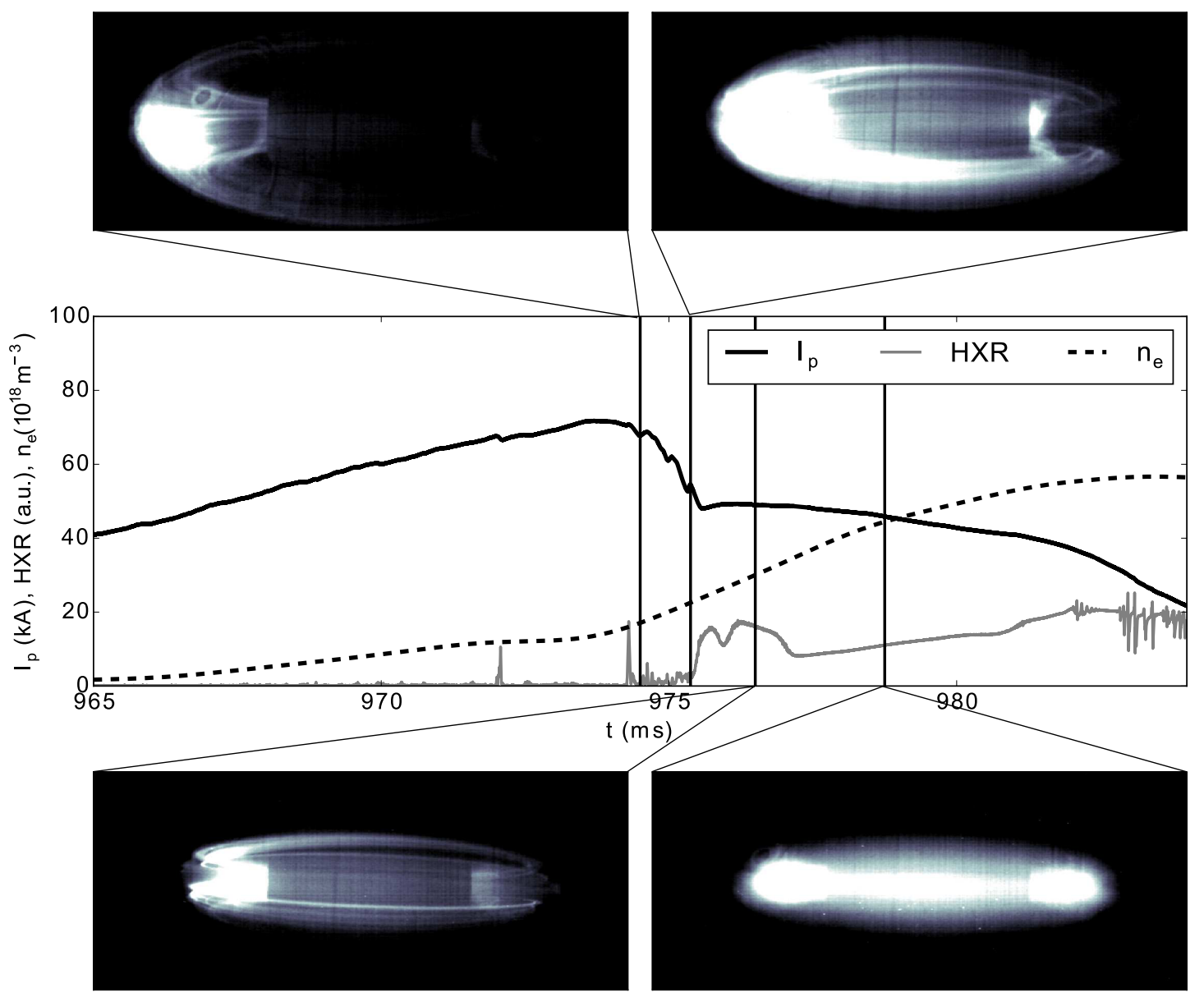

Figure 12. Time evolution of plasma current $I_{p}$, electron density $n_{e}$ and HXR signal during an Ar-induced disruption at the COMPASS tokamak with four frames from the fast camera capturing plasma at the indicated time stamps.

\section{Conclusions}

The runaway electron losses have been studied on COMPASS in both quiescent low density discharges and Ar-induced disruptions with runaway electron beam generation. These experiments benefit from the experience with the influence of initial fuelling on the RE generation in the current ramp-up phase of the discharge. The effect of the magnetic field perturbations on the RE losses in the COMPASS tokamak was studied using a rich set of magnetic diagnostics and HXR scintillation detectors. It was found that REs are extremely sensitive to any magnetic field perturbation as the RE losses are modulated in a wide range of frequencies (hundreds of $\mathrm{Hz}$ to tens of $\mathrm{kHz}$ ). In the flattop of the low density discharges the effect of periodic RE losses to the OMP limiter caused by magnetic island rotation was observed and from the identified phase order it was concluded that maximum of the losses occurs when the $\mathrm{O}$ point of the island passes the limiter. The amplitudes of losses were found to be considerably lower in some discharges 
with magnetic islands - REs seem to be confined by them. Furthermore, it was observed that the power source oscillations cause periodic HXR intensity peaks with a frequency dependent also on the edge safety factor. Unlike the simple effect that can be directly related to the oscillations of magnetic axis position caused by the power sources, the higher frequency of HXR peaks seems to result from an interaction between the power source oscillations and plasma MHD instabilities.

The link between the MHD perturbations and losses of RE was also observed during the MGI disruptions, namely in those accompanied by the RE beam generation. The beam generation has been achieved despite the low toroidal field in COMPASS. Observation of thin filaments in the early beam plateau phase, which corresponds to a final stage of the formation of the beam, is accompanied by increased HXR losses and rather small magnetic turbulence bursts. In agreement with the previous studies, the disruptions with RE beam generation are generally characterised by a smaller level of high frequency magnetic fluctuations. However, no clear threshold magnetic perturbation magnitude has been found in this respect. The MHD dynamics of the post-disruption beam itself merits further investigation on COMPASS, with results contributing, among others, to benchmarking of the advanced RE models. Importantly, the influence of transient MHD phenomena on the RE losses has to be taken into account in order to quantify the RE related risks and to verify the mitigation schemes for ITER.

\section{References}

[1] Hender T. C. 2007 Nucl. Fusion 47 (6), S128-S202

[2] Zeng L. et al., 2013 Phys. Rev. Lett. 110235003

[3] Sommariva C. et al. 2016 Modeling Runaway Electron dynamics in realistic fields from 3D nonlinear MHD disruption simulations 43rd EPS Conf. on Plasma Physics (Leuven, Belgium) $\mathrm{P} 2.006$

[4] Czarny O. and Huysmans G. 2008 J. Comp. Phys. 227 (16) 7423-7445

[5] Panek R. et al. 2016 Plasma Phys. Control. Fusion 58014015

[6] Panek R. et al. 2016 Czech. J. Phys., 56 B125-B137

[7] Mlynar J. et al., 2015 Effects of Plasma Control on Runaway Electrons in the COMPASS Tokamak 42nd EPS Conf. on Plasma Physics (Lisbon, Portugal) P4.102

[8] Ficker O. et al. 2016 58th Ann. Meeting APS DPP (San Jose, USA) G10.101

[9] Vlainic M. et al. 2015 J. Plasma Phys. 81475810506

[10] Weinzettl V. et al. 2011 Fusion Eng. Des. 86 1227-1231

[11] Imrisek M. et al. 2014 Ref. Sci. Instrum. 83 11E433

[12] Klimanov I. et al. 2011 Plasma Phys. Control. Fusion 49 L1 - L6

[13] Weinzettl V. et al. 2013 Experimental evidence of neoclassical tearing modes on COMPASS tokamak 40th EPS Conf. on Plasma Physics (Espoo, Finland) P5.143

[14] Rabinski M. et al. 2011 Voprosy Atomnoj Nauki i Tekhniki 41 164-166

[15] Causa F. et al. 2015 42nd EPS Conf. on Plasma Physics (Lisbon, Portugal) O4.134

[16] Havlicek, J. et al. 2014 Annual Conference of Doctoral Students WDS (Prague, Czech Republic)

[17] Vondracek, P., et al. Submitted to Fusion Eng. Des. (2016)

[18] Granetz R. S. et al. 2014 Phys. Plasmas 21072506

[19] Moyer, R.A. et al. 2014 Impact of Core Modes, Islands, and Instabilities on Runaway Electron Confinement Proc. 25th IAEA FEC (St. Petersburg, Russia) General Atomics Report GAA27773 
[20] Reux C et al. 2015 Nucl. Fusion 55129501

[21] Plyusnin V. et al. 2016 Comparison of Runaway Electron Generation Parameters in Small, Medium-sized and Large Tokamaks: A Survey of Experiments in COMPASS, TCV, ASDEXUpgrade and JET Proc. 26th IAEA FEC (Kyoto, Japan)

[22] Martin, P. et al. 2016 Physics, control and mitigation of disruptions and runaway electrons in the EUROfusion Medium Size Tokamaks science programme Proc. 26th IAEA FEC (Kyoto, Japan)

[23] Papp, G. et al. 2016 Runaway electron generation and mitigation on the European medium sized tokamaks ASDEX Upgrade and TCV Proc. 26th IAEA FEC (Kyoto, Japan)

[24] Helander P. et al. 2000 Phys. Plasmas, 7 (10) 4106 - 4111

[25] Vlainic M. et al. 2015 Synchrotron radiation from runaway electrons in the COMPASS tokamak 42nd EPS Conference on Plasma Physics in Lisbon 42nd EPS Conf. on Plasma Physics (Lisbon, Portugal) P4.102

[26] Esposito B. et al. 2016 First experimental results of runaway beam control in TCV Proc. 26th IAEA Fusion Energy Conference (Kyoto, Japan)

[27] Carnevale D. et al. 2016 Analysis of runaway beam suppression experiments in FTU Proc. 26th IAEA Fusion Energy Conference (Kyoto, Japan)

[28] Martin-Solis J. R. et al. 2016 Current profile shape effects on the formation and termination of runaway beams in tokamak disruptions and implications for ITER Proc. 26th IAEA FEC (Kyoto, Japan)

[29] Plyusnin V. V. et al. 2008 Rev. Sci. Instrum. 79 (10) 10F505

[30] Abdullaev S. S. et al. 2015 J. Plasma Phys. 81

[31] Hollmann E. M. et al. 2013 Nucl. Fusion 53083004 (15pp)

[32] Coda S. et al. Submitted to Nucl. Fusion (2016)

\section{Acknowledgments}

This work has been supported by MEYS projects LG14002 and LM2015045 and carried out within the framework of the EUROfusion Consortium. It has also received funding from the Euratom research and training programme 2014-2018 under grant agreement No 633053 with the Co-fund by MEYS project number 8D15001. The views and opinions expressed herein do not necessarily reflect those of the European Commission. 